\title{
Spin-polarized current versus stray field in a perpendicularly magnetized superconducting spin switch
}

\author{
A. Singh, C. Sürgers, ${ }^{\text {a) }}$ and R. Hoffmann \\ Physikalisches Institut, Universität Karlsruhe, D-76128 Karlsruhe, Germany \\ H. v. Löhneysen \\ Physikalisches Institut, Universität Karlsruhe, D-76128 Karlsruhe, Germany and \\ Forschungszentrum Karlsruhe, Institut für Festkörperphysik, D-76021 Karlsruhe, Germany \\ T. V. Ashworth and N. Pilet \\ Department of Physics, University of Basel, Klingelbergstrasse 82, CH-4056 Basel, Switzerland \\ H. J. Hug \\ Department of Physics, University of Basel, Klingelbergstrasse 82, CH-4056 Basel, Switzerland and \\ EMPA Materials Science and Technology, Überlandstrasse 129, CH-8600 Dübendorf, Switzerland
}

(Received 18 June 2007; accepted 14 September 2007; published online 10 October 2007)

In superconducting $\mathrm{Co} / \mathrm{Pt}-\mathrm{Nb}-\mathrm{Co} / \mathrm{Pt}$ multilayers with perpendicular magnetic anisotropy, the superconducting transition temperature $T_{c}$ is considerably higher in the parallel (P) orientation of the two $\mathrm{Co} / \mathrm{Pt}$ magnetizations compared to the antiparallel (AP) orientation. Low temperature magnetic-force microscopy reveals that the individual ferromagnetic layers are uniformly magnetized in both configurations and do not exhibit a magnetic stray field arising from a multidomain state. We also demonstrate that the difference $\Delta T_{c}=T_{c}^{\mathrm{P}}-T_{c}^{\mathrm{AP}}$ between both $T_{c}$ values is enhanced at higher transport currents due to the increasing imbalance of spin-polarized charge carriers in the superconducting $\mathrm{Nb}$ layer. Hence, the spin switch can be controlled by the transport current in future superconducting devices. () 2007 American Institute of Physics. [DOI: $10.1063 / 1.2794424]$

In magnetoelectronic or spintronic devices, injection, accumulation, and reflection of spin-polarized charge carriers play important roles. ${ }^{1}$ Ferromagnet $(\mathrm{F}) /$ normal metal $(\mathrm{N}) / \mathrm{F}$ spin valves utilizing the giant magnetoresistance (GMR) effect are used for data storage applications. Recently, a GMR effect was observed in superconductor $(\mathrm{S}) / \mathrm{F}$ multilayers (ML), ${ }^{2,3}$ where spin-polarized carriers injected from $\mathrm{F}$ into $\mathrm{S}$ strongly suppress the critical current density $J_{c}{ }^{4}$ In a FSF spin switch, the transition temperature $T_{c}$ of an $\mathrm{S}$ layer sandwiched between two $\mathrm{F}$ layers depends on their relative inplane magnetization orientation-parallel $(\mathrm{P})$ or antiparallel (AP)—with $T_{c}^{\mathrm{P}}<T_{c}^{\mathrm{AP}} \cdot{ }^{5,6}$ However, the inverse behavior $T_{c}^{\mathrm{P}}$ $>T_{c}^{\mathrm{AP}}$ has also been reported for various systems, including FSF triple layers with perpendicular magnetic anisotropy (PMA).

In order to explain $T_{c}^{\mathrm{P}}>T_{c}^{\mathrm{AP}}$ in S/F-ML two conflicting models have been proposed. In a model based on the GMR effect the spin-polarized charge carriers experience an enhanced spin-dependent reflection at the $\mathrm{F} / \mathrm{S}$ interface in the AP state. Hence, they will accumulate in the S layer which gives rise to a reduction of the superconducting energy gap provided that the thickness of $\mathrm{S}$ is smaller than the spin diffusion length $\left(\lambda_{\text {sd }} \approx 250 \AA\right.$ for $\left.\mathrm{Nb}\right){ }^{2,3,8}$ In this case, it is important to confirm that no finite domains generating magnetic stray fields are formed due to interlayer magnetostatic coupling. ${ }^{9}$ Moreover, if spin-dependent scattering plays a dominant role, the difference $\Delta T_{c}=T_{c}^{\mathrm{P}}-T_{c}^{\mathrm{AP}}$ should increase with increasing current flowing perpendicularly to the interfaces, i.e., with increasing number of spin-polarized quasiparticles injected from $\mathrm{F}$ into $\mathrm{S}$. This picture is also valid for

\footnotetext{
${ }^{a)}$ Electronic mail: christoph.suergers@pi.uka.de
}

a current-in-plane configuration, where only a fraction of the charge carriers will cross the interfaces between the $\mathrm{S}$ and $\mathrm{F}$ layers.

A second model is based on a stray-field based mechanism, where the $\mathrm{F}$ layer forms a multidomain state near its coercive field ${ }^{10-12}$ and the $S$ layer experiences a perpendicular stray field due to the Bloch or Néel walls, which suppresses the superconducting properties by Lorentz-force induced motion of vortices. ${ }^{11,12}$ However, critical-field measurements of FS-ML suggest that the stray field of the magnetized $F$ films is close to zero except at the sample edges. ${ }^{13,14}$

In this paper we address these issues by studying a $\mathrm{Nb}$ film that is sandwiched between a bottom (b) and top ( $\mathrm{t}$ ) ferromagnetic Co/Pt ML with PMA. We investigated the different magnetic states by low-temperature-magnetic force microscopy and the effect of current on the magnitude of $\Delta T_{c}$. Samples of $(4 \AA \mathrm{Co} / 10 \AA \mathrm{Pt})_{6}^{b} / 10 \AA \mathrm{Pt} / d_{\mathrm{Nb}} /$ $20 \AA \mathrm{Pt} /(6 \AA \mathrm{Co} / 10 \AA \mathrm{Pt})_{4}^{t}$ (subscripts denote number of repeat units, $d_{\mathrm{Nb}}$ : thickness of $\mathrm{Nb}$ film) were deposited by electron-beam evaporation. ${ }^{15}$ The electrical resistance $R$ was measured on stripe-shaped samples $\left(7 \times 3 \mathrm{~mm}^{2}\right)$ with the current in the plane. Magnetization measurements were performed in a vibrating sample magnetometer above $T_{c}$ in perpendicularly applied field. Magnetic domain images were taken at low temperatures in a home-built magnetic force microscope (MFM) ${ }^{16}$ A cantilever with a spring constant of $0.2 \mathrm{~N} / \mathrm{m}$ and a resonance frequency of $39.935 \mathrm{kHz}$ was used. Its high-aspect ratio tip was coated on one side with a 3 -nm-thick cobalt layer. The tip magnetization was found to flip reproducibly between up and down states at fields of $\pm 120 \mathrm{mT}$. All MFM measurements were taken at a constant 

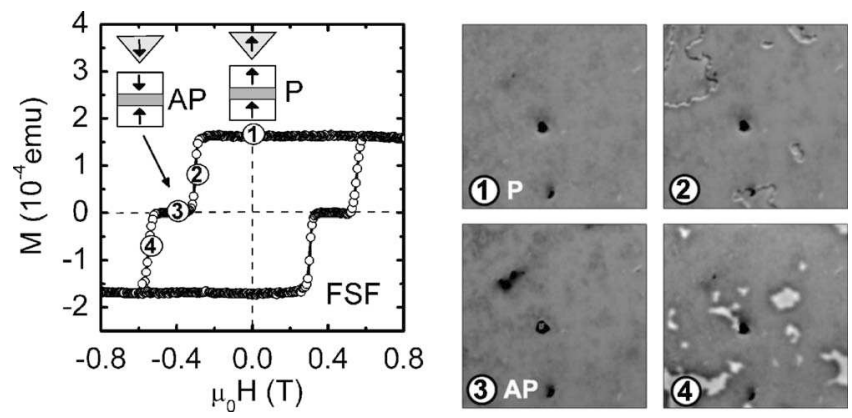

FIG. 1. Left: hysteresis loop $M(H)$ at $T=8 \mathrm{~K}$ of a FSF sample with $d_{\mathrm{Nb}}$ $=200 \AA\left(T_{c}=4.835 \mathrm{~K}\right)$. The P or AP configurations and the MFM tip magnetization are schematically indicated in the insets (gray layer: $\mathrm{Nb}$ ). Right: $3 \times 3 \mu \mathrm{m}^{2} \mathrm{MFM}$ images taken at $10 \mathrm{~K}$ for various magnetic fields. 1: zero field, 2: $-300 \mathrm{mT}, 3:-400 \mathrm{mT}$, and 4: $-540 \mathrm{mT}$. The MFM images are all represented with the same contrast of $\pm 4.5 \mathrm{~Hz}$.

average tip-sample distance, in the dynamic variable frequency mode, which determines the shift $\Delta f$ of the cantilever resonance frequency $f_{0}$.

The magnetic hysteresis loop $M(H)$ of a FSF sample with $d_{\mathrm{Nb}}=200 \AA$ shown in Fig. 1 is characteristic for ferromagnetic films with PMA. ${ }^{13,14}$ The different substrate temperatures used during growth of the ML yield a higher coercive field $\mu_{0} H_{c}^{b}$ for the bottom ML than $\mu_{0} H_{c}^{t}$ for the top ML. Due to the difference of $\approx 200 \mathrm{mT}$ between the coercive fields, a $\mathrm{P}$ orientation can be easily achieved by increasing the field up to $+1 \mathrm{~T}$ just above $T_{c}$ and subsequently switching the field off. ${ }^{7}$ In order to obtain the AP orientation, a reverse field between $-\mu_{0} H_{c}^{b}$ and $-\mu_{0} H_{c}^{t}$ was applied after saturation above $T_{c}$ and after that the field was switched off.

Figure 1 shows the magnetic domain structure acquired with the MFM at $T=10 \mathrm{~K}$ at fields indicated in the $M(H)$ loop. The two black spots (negative frequency shift) in the image are due to topographical features leading to larger attractive van der Waals forces due to the decreased local tipsample distance. Note that these features occur in all MFM images acquired on this sample position and hence demonstrate that all images were acquired at exactly the same position.

In zero field, no domains are visible in the MFM image, confirming that both $\mathrm{F}$ layers remain saturated. At a field of $-300 \mathrm{mT}$ a few irregularly shaped domains become visible. The observed MFM contrast is mainly due to the domain walls, particularly for the micron-sized domain in the upper left of the image. This confirms that the distance between the tip and the domains is small, i.e., that these domains are located in the top F layer. Note that the magnetization of the MFM tip is now flipped into the down state. At $-400 \mathrm{mT}$ no domains are observed, confirming that both $\mathrm{F}$ layers are saturated but with an antiparallel orientation of their magnetizations. At $-540 \mathrm{mT}$, domains are again visible but appear as irregular areas of a rather homogenous white contrast (domain instead of domain-wall contrast). This confirms that these domains are magnetized along the initial up direction and are located in the bottom F layer. From the absence of MFM contrast in the P and AP states we conclude that no stray fields exist in these states (well away from the edges of the sample).

To further support an underlying GMR mechanism, the dependence of the resistive superconducting transition $R(T)$ on the transport current $I$ was measured [see Fig. 2(a)]. The main result is that the difference between the $T_{c}$ values in the $\mathrm{P}$ and AP states, taken at $50 \%$ of the residual resistance at $10 \mathrm{~K}$, increases with current (or current density $J$ ). This can be understood because the transport current induces a diffusion of nonequilibrium spin-polarized charge carriers from $\mathrm{F}$ to $\mathrm{S}$ thus reducing $T_{c}$. In addition, the width of each transition increases with current. For $I=30$ and $100 \mu \mathrm{A}$ the width is larger for the AP state than for the P state. Figure 2(b) shows that the relative difference $\Delta T_{c} / T_{c}^{\mathrm{P}}$ increases almost linearly with $I$ for small $I$ and eventually saturates. This is attributed to the induced diffusion of spin-polarized quasiparticles from $\mathrm{F}$ into $\mathrm{S}$ which is increasing with $I$. An effect due to a depinning of stray-field induced vortices leading to dissipation can be ruled out since the MFM images do not show large magnetic domains. Moreover, such a stray-field effect should be independent of the charge-carrier transmission across the F/S interface and therefore should also occur in samples with an insulating barrier between $\mathrm{F}$ and $\mathrm{S}$. This is not observed as discussed below. Figure 2(b) further shows that for a fixed current $I$ the magnitude of $\Delta T_{c} / T_{c}^{\mathrm{P}}$ increases with decreasing $\mathrm{Nb}$ thickness, indicating that the spinpolarized charge carriers are effective in breaking of Cooper pairs only within the spin-diffusion length $\lambda_{\text {sd. }}{ }^{7}$ For $d \gtrsim \lambda_{\text {sd }}$ the sample is in an inhomogeneous state, with $T_{c}$ not being changed appreciably in the central part of the $S$ layer. This may be the reason for the broadening of the transition width at higher $I$.

Consequently, the critical current also depends on the magnetic state and is somewhat higher for the $\mathrm{P}$ state than for the AP state, as shown in Fig. 2(c). This again proves the existence of a true AP state with saturated F layers, because a multidomain state would give rise to an increased pinning of induced vortices and therefore to a higher critical current in the AP state ${ }^{17}$ contrary to what is observed. Hence, our results are in agreement with spin-imbalance theory. ${ }^{18}$

Finally, an analogous investigation was performed on FISIF samples, where I is a 25 - $\AA$-thick insulating $\mathrm{SiO}_{2}$ layer sandwiched between $\mathrm{F}$ and $\mathrm{S}$ layers. This I layer strongly reduces the proximity effect and the transmission of charge carriers between $\mathrm{F}$ and $\mathrm{S}$. The $M(H)$ loop of a FISIF sample shown in Fig. 3(a) is similar to that of the FSF sample. The resistive transitions plotted in Fig. 3(b) are very sharp and $T_{c}$
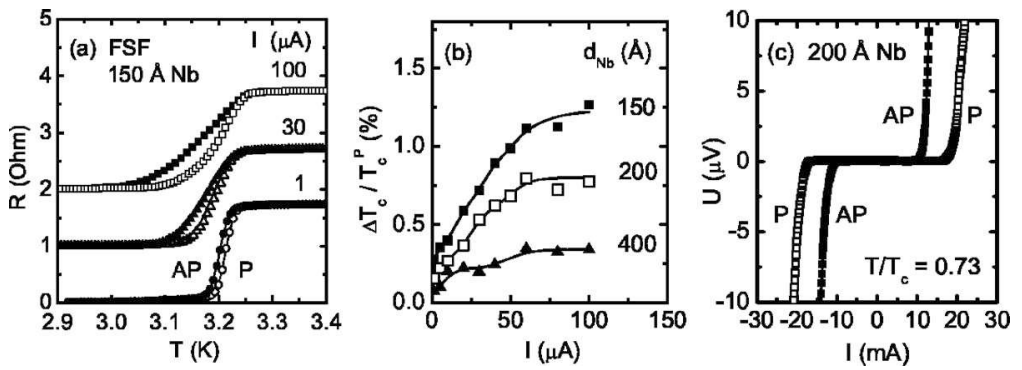

FIG. 2. (a) Resistive superconducting transitions for an FSF triple layer in the AP state (closed symbols) and P state (open symbols) for different in-plane currents $I$. Each curve is shifted upward by $1 \Omega$ for clarity. (b) The relative $T_{c}$ difference $\Delta T_{c} / T_{c}^{\mathrm{P}}=\left(T_{c}^{\mathrm{P}}-T_{c}^{\mathrm{AP}}\right) / T_{c}^{\mathrm{P}}$ vs current $I$ for different $d_{\mathrm{Nb}}$. (c) Voltage-current characteristics at $T / T_{c}=0.73$. 

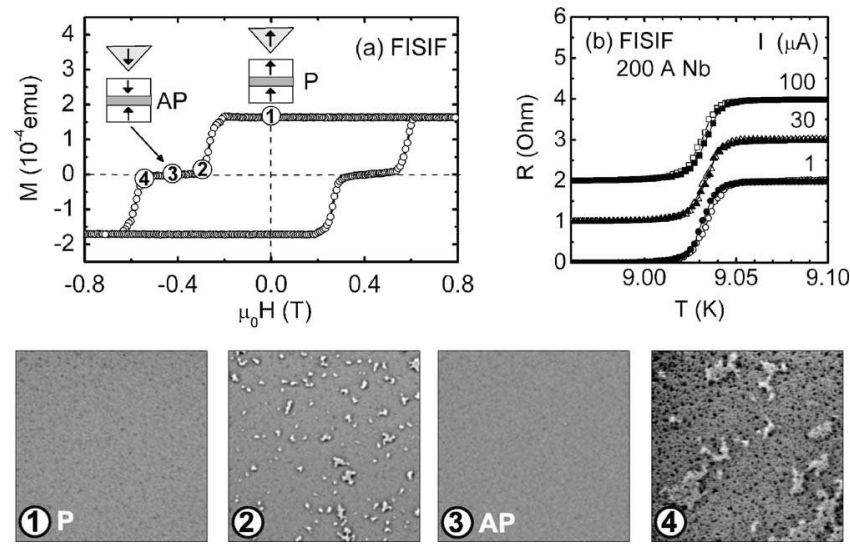

FIG. 3. (a) $M(H)$ at $T=10 \mathrm{~K}$ of a FISIF sample with $d_{\mathrm{Nb}}=200 \AA\left(T_{c}\right.$ $=9.031 \mathrm{~K}$ ). (b) $R(T)$ in the AP state (closed symbols) and P state (open symbols) for different in-plane currents $I$. Each curve is shifted upward by $1 \Omega$ for clarity. Bottom: $5 \times 5 \mu \mathrm{m}^{2} \mathrm{MFM}$ images taken at $11 \mathrm{~K}$ for various magnetic fields. 1: zero field, 2: $-305 \mathrm{mT}, 3:-425 \mathrm{mT}$, and 4: $-540 \mathrm{mT}$. The MFM images are all represented with the same contrast of $\pm 2 \mathrm{~Hz}$.

is close to the value for bulk $\mathrm{Nb}$ due to the suppression of the proximity effect and the reduced transmission of charge carriers across the metal interfaces. More importantly, $T_{c}$ is independent of the magnetic orientation as well as of the transport current.

Figure 3 shows the MFM images obtained on this sample. Again no domains are visible in the $\mathrm{P}$ and AP states (panels 1 and 3). The dark features that are visible in all images but particularly in image 4 , that was taken at a slightly smaller tip-sample distance, are due to the topography of the sample. Note that these features appear at the same locations in all four images, hence all four images were taken at the same sample location. Domains are visible only when either the top or the bottom F layer switches its magnetization from the initial up into the down state, but is not yet saturated.

We conclude that FSF triple layers with PMA have uniformly magnetized $\mathrm{F}$ layers in the $\mathrm{P}$ and $\mathrm{AP}$ states. The magnitude of superconducting spin-switch effect $T_{c}^{\mathrm{P}}>T_{c}^{\mathrm{AP}}$ can be enhanced by increasing the spin imbalance in the supercon- ductor, i.e., by injecting a larger number of spin-polarized charge carriers. Such a hybrid structure consisting of S layers and $\mathrm{F}$ layers with PMA can lead to future devices where the spin-polarized current can modulate superconductivity.

This work was supported by the Alexander von Humboldt Foundation (A.S.), the DFG Center for Functional Nanostructures, the Landesstiftung Baden-Württemberg, the Swiss National Science Foundation, and the NCCR Nanoscale Science. We thank D. Beckmann and J. Aarts for valuable discussions.

${ }^{1}$ I. Žutić, J. Fabian, and S. Das Sarma, Rev. Mod. Phys. 76, 323 (2004).

${ }^{2}$ V. Peña, Z. Sefrioui, D. Arias, C. Leon, J. Santamaria, J. L. Martinez, S. G. E. te Velthuis, and A. Hoffmann, Phys. Rev. Lett. 94, 057002 (2005).

${ }^{3}$ C. Visani, V. Peña, J. Garcia-Barriocanal, D. Arias, Z. Sefrioui, C. Leon, J. Santamaria, N. M. Nemes, M. Garcia-Hernandez, J. L. Martinez, S. G. E. te Velthuis, and A. Hoffmann, Phys. Rev. B 75, 054501 (2007).

${ }^{4}$ S. Sarkar, P. Raychaudhuri, P. K. Mal, A. R. Bhangale, and R. Pinto, J. Appl. Phys. 89, 7502 (2001).

${ }^{5}$ L. R. Tagirov, Phys. Rev. Lett. 83, 2058 (1999).

${ }^{6}$ A. I. Buzdin, A. V. Vedyayev, and N. V. Ryzhanova, Europhys. Lett. 48, 686 (1999).

${ }^{7}$ A. Singh, C. Sürgers, and H. v. Löhneysen, Phys. Rev. B 75, 024513 (2007).

${ }^{8}$ A. Y. Rusanov, S. Habraken, and J. Aarts, Phys. Rev. B 73, 060505(R) (2006).

${ }^{9}$ V. Baltz, A. Marty, B. Rodmacq, and B. Dieny, Phys. Rev. B 75, 014406 (2007).

${ }^{10}$ R. Steiner and P. Ziemann, Phys. Rev. B 74, 094504 (2006).

${ }^{11}$ C. Bell, S. Turşucu, and J. Aarts, Phys. Rev. B 74, 094504 (2006).

${ }^{12}$ D. Stamopolous, E. Manios, and M. Pissas, Phys. Rev. B 75, 184504 (2007).

${ }^{13}$ M. Lange, M. J. Van Bael, and V. V. Moshchalkov, Phys. Rev. B 68, 174522 (2003)

${ }^{14}$ W. Gillijns, A. Yu. Aladyshkin, M. Lange, M. J. Van Bael, and V. V. Moshchalkov, Phys. Rev. Lett. 95, 227003 (2005).

${ }^{15}$ A. Singh, C. Sürgers, M. Uhlarz, S. Singh, and H. v. Löhneysen, Appl. Phys. A: Mater. Sci. Process. 89, 593 (2007).

${ }^{16}$ H. J. Hug, B. Stiefel, P. J. A. van Schendel, A. Moser, S. Martin, and H.-J. Güntherodt, Rev. Sci. Instrum. 70, 3625 (1999).

${ }^{17}$ D. B. Jan, J. Y. Coulter, M. E. Hawley, L. N. Bulaevskii, M. P. Maley, Q. X. Jia, B. B. Maranville, F. Hellman, and X. Q. Pan, Appl. Phys. Lett. 82, 778 (2003)

${ }^{18}$ S. Takahashi and S. Maekawa, Phys. Rev. B 67, 052409 (2003). 